\title{
A Poor-Quality Generic Drug for the Treatment of Visceral Leishmaniasis: A Case Report and Appeal
}

\section{Thomas P. C. Dorlo ${ }^{1,2 *}$, Teunis A. Eggelte ${ }^{1}$, Gerard J. Schoone ${ }^{3}$, Peter J. de Vries ${ }^{1}$, Jos H. Beijnen ${ }^{2}$}

1 Division of Infectious Diseases, Academic Medical Center, University of Amsterdam, Amsterdam, The Netherlands, 2 Department of Pharmacy \& Pharmacology, Slotervaart Hospital / the Netherlands Cancer Institute, Amsterdam, The Netherlands, 3 Department of Parasitology, KIT Biomedical Research, Amsterdam, The Netherlands

A generic miltefosine pharmaceutical product containing no active pharmaceutical ingredient for the treatment of visceral leishmaniasis emerged in Bangladesh for use in the national elimination programme. Poor-quality drugs for the treatment of this fatal neglected tropical disease are life-threatening for the vulnerable patients using them but also have a devastating impact on public health and elimination programmes targeting this disease. National drug regulators should take responsibility and ensure without any concessions that procured drugs for neglected tropical diseases, either innovator or generic, adhere to international standards for drug quality and safety.

\section{Introduction}

Proper chemotherapy is pivotal in the management of visceral leishmaniasis (VL, also known as kala-azar); without an effective treatment this neglected parasitic disease is inevitably fatal [1]. Nevertheless, the few new and safer but more expensive treatment options that were developed in the past decade (i.e., liposomal amphotericin B and miltefosine) remain largely out of reach of the affected rural population who are most in need, mainly the poorest of the poor [2-4]. Miltefosine, an alkylphosphocholine drug, is an essential drug in the management of $\mathrm{VL}$ as it is the first effective oral treatment option with a reasonable safety profile [5]. Oral miltefosine allows the treatment of VL patients without an extended period of hospital admission and thus puts fewer demands on both patients and health services $[6,7]$. Miltefosine is currently preferred for implementation in national VL elimination programmes [8], although the burden of high treatment costs incites the exploration of possibilities for a generic miltefosine product [9]. Unfortunately, the precarious position of $\mathrm{VL}$ patients was recently jeopardized as patients in Bangladesh were confronted with a new threat: the emergence of a new miltefosine product containing no active pharmaceutical ingredient $[10,11]$.

\section{Case Description}

Together with Nepal and India, the government of Bangladesh has committed to eliminate VL by 2015, supported by the World Health Organization (WHO) $[8,12]$. Interventions in this VL elimination programme comprise active case surveillance and implementation of vector control management strategies, but also improvement of the availability of appropriate drugs [13]. Oral miltefosine was recommended for this strategy and was therefore registered in Bangladesh [14]. Nevertheless, problems relating to its procurement and supply prohibited accessibility of this treatment and required a less costly alternative. Local procurement of miltefosine was therefore sought and a generic product supposedly containing miltefosine named "Miltefos" was manufactured by a local company for use in the Bangladeshi national elimination programme for VL. In early 2008, "Miltefos" was implemented as first-line therapy for VL in Bangladesh [8]. Although official numbers remain absent, reports from the field indicated abnormal "poor responses in hundreds of patients" after the use of "Miltefos" [8,10], thereby clearly contradicting high historic efficacy rates $(\sim 95 \%)$ of miltefosine in VL in nearby Indian and Nepalese provinces [15]. Therefore bioequivalence studies were planned to compare the local generic "Miltefos" product to the innovator "Impavido" product (Paladin Labs); however, the validity of the underlying assumption of pharmaceutical equivalence had to be established first. For this reason drug samples were sent from Bangladesh to our lab to analyze them for their miltefosine content. To our best knowledge, only two different "Miltefos" batches were produced and distributed in Bangladesh and representative drug samples from both these batches of "Miltefos" with respective label claims of "10 mg miltefosine" and " $50 \mathrm{mg}$ miltefosine" (Figure 1) were analyzed. A platform of analytical techniques was developed of which the methodology is described in more detail elsewhere [11]. A high-performance liquid chromatography coupled to tandem mass spectrometry (LCMS/MS) method validated for the detection of miltefosine with a lower limit of quantitation of $4 \mathrm{ng} / \mathrm{ml}$ in human plasma immediately revealed that no miltefosine could be identified in methanol extracts of any of the "Miltefos" capsules, while this method allowed the irrefutable identification and quantification of miltefosine in the "Impavido" capsules [16]. This absence of miltefosine was confirmed in the crude "Miltefos" capsule contents by Fourier transform infrared spectroscopy and a new, simple, and rapid colorimetric test for miltefosine with a lower limit of detection of at least $12.5 \mu \mathrm{g} / \mathrm{ml}$ [11]. Near-infrared spectroscopy even allowed us to confirm this finding without opening the capsules. Further tests using mass spectrometry could not identify any other

Citation: Dorlo TPC, Eggelte TA, Schoone GJ, de Vries PJ, Beijnen JH (2012) A Poor-Quality Generic Drug for the Treatment of Visceral Leishmaniasis: A Case Report and Appeal. PLoS Negl Trop Dis 6(5): e1544. doi:10.1371/ journal.pntd.0001544

Editor: Marleen Boelaert, Institute of Tropical Medicine, Belgium

Published May 29, 2012

Copyright: (c) 2012 Dorlo et al. This is an open-access article distributed under the terms of the Creative Commons Attribution License, which permits unrestricted use, distribution, and reproduction in any medium, provided the original author and source are credited.

Funding: No direct funding was received for this study. The authors were personally salaried by their institutions during the period of writing (though no specific salary was set aside or given for the writing of this paper).

Competing Interests: The authors have declared that no competing interests exist.

* E-mail: t.p.dorlo@amc.nl 


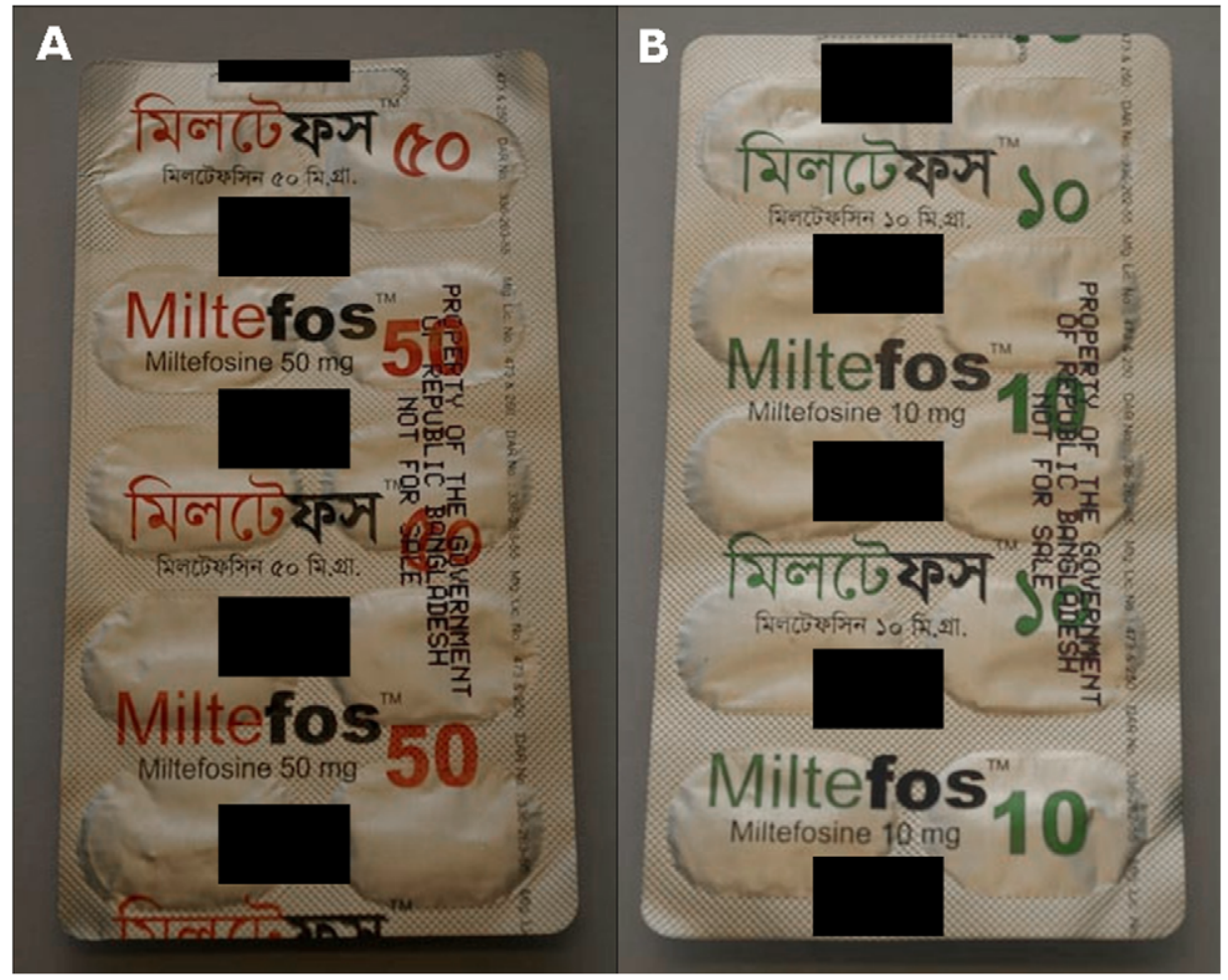

Figure 1. "Miltefos" blister packs. Backs of blister package "Miltefos, Miltefosine 50 mg" (A) and "Miltefos, Miltefosine $10 \mathrm{mg}$ " (B), respectively. The name and logo of the manufacturer have been obscured.

doi:10.1371/journal.pntd.0001544.g001

compound, including any possible degradation products of miltefosine, in the "Miltefos" capsule contents besides the common excipients lactose and microcrystalline cellulose [11].

To confirm these findings and to ensure that the pharmaceutical product under investigation was the same product that was being used by Bangladeshi VL patients, a second representative sample of "Miltefos" specimens was collected directly in the field in Bangladesh at a health centre where VL patients were under treatment with "Miltefos" at that time. Furthermore, whole blood samples from five VL patients who were still under treatment with "Miltefos" and the exact blisters that had been used to treat these patients were collected simultaneously. Both these blood and drug specimens were transported to our lab in sealed and signed envelopes to ensure the integrity of the parcel in transit. Case record forms and signed statements by the doctors of these patients indicating the origin and authenticity of the samples were included. The same analytical techniques as mentioned above reconfirmed the previous results: no miltefosine could be identified in these "Miltefos" batches, nor any other active pharmaceutical ingredient. Storage conditions in Bangladesh or during transport are not expected to have been of any influence, given miltefosine's excellent stability profile, also in humid and hot conditions [17]. Moreover, no miltefosine could be detected in the whole blood samples of the patients to whom these "Miltefos" capsules were administered. All these patients were already several days on "Miltefos" treatment and should have accumulated substantial miltefosine blood concentrations because of the extremely slow elimination of miltefosine from the body [18]. A real-time reversetranscriptase PCR (qRT-PCR) targeting the Leishmania $18 \mathrm{~S}$ ribosomal RNA performed on blood of these patients confirmed that they were suffering from VL, indicating the authenticity of the received samples [19]. Following these findings, clinical care of the patients was changed to treatment with intravenous sodium antimony gluconate. Eventually, "Miltefos" was removed from all patient care regimens throughout Bangladesh and intravenous sodium antimony gluconate was re-introduced as first-line therapy for VL in Bangladesh [8].

\section{Discussion}

This case demonstrates that despite existing international regulations for quality assurance of medicines, poor-quality drugs can be distributed through a nationwide treatment programme in a resource-poor country and reach patients suffering from a fatal neglected tropical disease whose survival depends on good-quality drugs. The control of anthroponotic VL in South Asia relies most importantly on early case detection and effective treatment, thereby reducing the human reservoir of disease $[20,21]$. The use of substandard or counterfeit drugs containing no or a subtherapeutic amount of active pharmaceutical ingredient not only severely jeopardizes the individual health of patients but also these control efforts for VL. This emphasizes the need for prioritizing the quality of antiinfective medicines that are being used in resource-poor countries to treat neglected tropical diseases and the development of simple and rapid methods to assess drug quality [22-24].

The poor-quality generic miltefosine product that we investigated can be considered as a substandard product according to WHO definitions, since it never contained any active pharmaceutical ingredient [25-27]. However, this product cannot directly be assumed to be a counterfeit as well, since a fraudulent motive for the mislabelling of this product cannot be inferred from our scientific 
investigation [28]. For VL, at least three previous cases of poor-quality drugs have been described in India, Nepal, and Sudan, all concerning antimonials and resulting in unacceptable toxicity and even death [29-31]. Poor-quality medicines, both substandard and counterfeit, constitute a major burden on the public health in resource-poor countries [10, $22,32,33]$. Inadequate local drug regulation and law enforcement combined with poor compliance of local pharmaceutical industry with good manufacturing practices (GMP) can lead to high rates of substandard drug production in resourcepoor countries, but also to a higher degree of deliberate counterfeiting activities $[22,34]$. Antimalarials have most extensively been reported as a victim of counterfeiting in resource-poor countries, with a focus on artesunate products containing no or only a subtherapeutic amount of active pharmaceutical ingredient [35-37]. Provision of free or inexpensive antimalarials has been mentioned as one of the possible solutions to this widespread problem, removing the financial incentive for counterfeiters [32]. It has also been suggested that the quality of drugs would be warranted if distributed "through official institutions" [4]. However, this case clearly underlines that provision of free anti-infectives - even through official institutions - does not necessarily imply that the problem of poor-quality anti-infectives in resourcepoor countries is resolved and strict monitoring of drug quality by the funding organizations or responsible government bodies is therefore key in any drug provision programme.

Without ignoring the ongoing debate on the various definitions of "substandard" and "counterfeit" [22-24,27,34,38], the emphasis in tackling poor-quality drugs should be on the safety of patients and public health, especially in resource-poor countries and certainly concerning the treatment of life-threatening diseases. Shifting the focus from intellectual property or trade issues towards public health and patients to define and combat poorquality drugs is therefore urgently needed [38]. Quality assurance of pharmaceutical products should be guaranteed by appropriate (government) bodies that have the specific mandate to protect individuals and public health, and should not be the responsibility of vulnerable individual patients themselves $[34,39]$. In resourcepoor countries it remains cumbersome to provide this protection, partly due to an urgent lack of both drug regulatory capacity and regional analysis laboratories for quality control of medicines. Previously, it has been shown that GMP compliant facilities can have parallel productions with lower standards for poorly regulated countries [34]. Quality assurance should therefore specifically extend to assessment of each manufacturing site and each product dossier according to the rigorous criteria set by the WHO for each individual drug that is being procured [40]. Several programmes have been initiated by the WHO (most notably the WHO Prequalification Programme) but separately also by other organizations (e.g., Médecins Sans Frontières) to facilitate qualification of drugs and access to technical expertise on this topic [34,41]. Unfortunately, the WHO Prequalification Programme selectively focuses on goodquality medicinal products relating to HIV/AIDS, tuberculosis, malaria, influenza, and reproductive health and has thus far ignored medicines against neglected diseases, although the availability of general technical expertise could help support regulatory authorities with limited capacities. This case indicates that it may be needed to extend the WHO Prequalification Programme to drugs for VL and other neglected diseases.

Availability and accessibility of regulatory information and technical expertise such as methods of analysis and identification for drugs for neglected diseases need to be prioritized. Currently, no monograph on miltefosine has been included in any of the major international pharmacopoeias, although the drug has been added to the WHO Model List of Essential Medicines and has received approval in Germany, which has a stringent regulatory authority $[42,43]$. International pharmacopoeias should cease to neglect drugs for neglected diseases to facilitate the quality control of these medicines and the production of generic pharmaceutical products. Furthermore, no formal approval of miltefosine or "Miltefos" could be traced in the publicly available information of the Bangladeshi drug regulatory authorities [44], despite WHO documents mentioning the registration of miltefosine in Bangladesh [14]. Full transparency of the current regulatory status of medicinal products is simply a prerequisite for the safe and effective use of drugs for neglected diseases, certainly in the resource-poor countries that are most affected.

Nevertheless, all these outstanding efforts can only be effective when national or regional drug regulators and procurement programmes in resource-poor countries are aware of the devastating impact of poor-quality drugs and decide to make use of all the (technical) resources that could be provided to them by international agencies. It must be emphasized that these recommendations certainly do not reject a priori the production or procurement of generic pharmaceutical products as a cheaper alternative for the treatment of neglected tropical diseases; however, no concessions should be tolerated in terms of assurance of quality and safety of these medicines, either innovator or generic.

\section{Conclusion}

VL patients belong to the poorest quintile of the population of resource-poor countries [3], which make them highly dependent on drug donations or drug provision by national elimination programmes for the treatment of their fatal disease. These extremely vulnerable patients deserve to be protected by national or regional drug regulators who should take responsibility by implementing the necessary precautions to prevent repetition of this poor-quality drug case. All procured VL drugs either used in the respective national VL elimination programmes, available over the counter, or used in clinical trials need to adhere to the WHO standards for quality and safety, irrespective of the country of origin of these drugs, whether they are innovator or generic medicines and whether they are intended to be used in resource-rich or resource-poor countries. Only under this condition can VL patients trust in a safe treatment, and national elimination programmes might have an impact on this typically neglected disease.

\section{Ethics Statement}

Written informed patient consent was obtained for the blood samples. Institutional review board approval was not required, since patients were not treated in the context of a study and the described procedures on the blood samples were performed to ensure that these patients received adequate treatment. 


\section{References}

1. Sengupta PC (1947) History of kala-azar in India. Indian Med Gaz 82: 281-286.

2. Sundar S, Murray HW (2005) Availability of miltefosine for the treatment of kala-azar in India. Bull World Health Organ 83: 394-395.

3. Boelaert M, Meheus F, Sanchez A, Singh SP, Vanlerberghe V, et al. (2009) The poorest of the poor: a poverty appraisal of households affected by visceral leishmaniasis in Bihar, India. Trop Med Int Health 14: 639-644.

4. den Boer M, Argaw D, Jannin J, Alvar J (2011) Leishmaniasis impact and treatment access. Clin Microbiol Infect 17: 1471-1477.

5. Sindermann H, Engel J (2006) Development of miltefosine as an oral treatment for leishmaniasis. Trans R Soc Trop Med Hyg 100 Suppl 1: S17-S20.

6. Meheus F, Balasegaram M, Olliaro P, Sundar S, Rijal S, et al. (2010) Cost-effectiveness analysis of combination therapies for visceral leishmaniasis in the Indian subcontinent. PLoS Negl Trop Dis 4: e818. doi:10.1371/journal.pntd.0000818.

7. Vanlerberghe V, Diap G, Guerin PJ, Meheus F, Gerstl S, et al. (2007) Drug policy for visceral leishmaniasis: a cost-effectiveness analysis. Trop Med Int Health 12: 274-283.

8. World Health Organization - Regional Office for South-East Asia (2010) Programme manager's meeting on elimination of Kala-azar in the SouthEast Asia Region, Faridabad, Haryana, India, 17-19 February 2009. Available: http://www. searo.who.int/LinkFiles/Kala_azar_Rec-09.pdf. Accessed 7 May 2012.

9. World Health Organization (WHO) (2010) WHO Technical Report Series 949: Annex 6 Costs of medicines in current use for the treatment of leishmaniasis. Available: http:// www.who.int/leishmaniasis/research/978_92_4_ 12_949_6_Annex6.pdf. Accessed 7 May 2012.

10. Senior K (2008) Global health-care implication of substandard medicines. Lancet Infect Dis 8: 666.

11. Dorlo TPC, Eggelte TA, de Vries PJ, Beijnen JH (2012) Characterization and identification of suspected counterfeit miltefosine capsules. Analyst 137: 1265-1274.

12. Bhattacharya SK, Sur D, Sinha PK, Karbwang J (2006) Elimination of leishmaniasis (kala-azar) from the Indian subcontinent is technically feasible \& operationally achievable. Indian J Med Res 123: 195-196.

13. Sundar S, Mondal D, Rijal S, Bhattacharya S, Ghalib H, et al. (2008) Implementation research to support the initiative on the elimination of kala azar from Bangladesh, India and Nepal-the challenges for diagnosis and treatment. Trop Med Int Health 13: 2-5.

14. World Health Organization - Regional Office for South-East Asia (2007) Regional Technical Advisory Group on Kala-azar Elimination - Report of the Second Meeting, Kathmandu, Nepal, 30 October-2 November 2006. Available: http:// searo.who.int/LinkFiles/Kala_azar_VBC-93.pdf. Accessed 7 May 2012.

15. Bhattacharya SK, Sinha PK, Sundar S Thakur CP, Jha TK, et al. (2007) Phase 4 trial of miltefosine for the treatment of Indian visceral leishmaniasis. J Infect Dis 196: 591-598.

16. Dorlo TPG, Hillebrand MJX, Rosing H, Eggelte TA, de Vries PJ, et al. (2008) Development and validation of a quantitative assay for the measurement of miltefosine in human plasma by liquid chromatography-tandem mass spectrometry. J Chromatogr B Analyt Technol Biomed Life Sci 865: 55-62.

17. German Drug Registration Authorities (2008) Impavido 10/50 mg Kapseln- Fachinformation. Available: http://www.pharmnet-bund.de/ dynamic/de/index.html. Accessed 15 February 2010.

18. Dorlo TPC, van Thiel PPAM, Huitema ADR, Keizer RJ, de Vries HJC, et al. (2008) Pharmacokinetics of miltefosine in Old World cutaneous leishmaniasis patients. Antimicrob Agents Chemother 52: 2855-2860.

19. van der Meide W, Guerra J, Schoone G, Farenhorst M, Coelho L, et al. (2008) Comparison between quantitative nucleic acid sequencebased amplification, real-time reverse transcriptase PCR, and real-time PCR for quantification of Leishmania parasites. J Clin Microbiol 46. 73-78.

20. Boelaert M, Griel B, Leeuwenburg J, Van Damme W, Le Ray D, et al. (2000) Visceral leishmaniasis control: a public health perspective. Trans R Soc Trop Med Hyg 94: 465-471.

21. Desjeux P (2004) Leishmaniasis: current situation and new perspectives. Comp Immunol Microbiol Infect Dis 27: 305-318.

22. Newton PN, Green MD, Fernández FM (2010) Impact of poor-quality medicines in the "developing" world. Trends Pharmacol Sci 31: 99-101.

23. Newton PN, Lee SJ, Goodman C, Fernández FM, Yeung S, et al. (2009) Guidelines for field surveys of the quality of medicines: a proposal. PLoS Med 6: e1000052. doi:10.1371/journal.pmed. 1000 052 .

24. Fernandez FM, Hostetler D, Powell K, Kaur H, Green MD, et al. (2011) Poor quality drugs: grand challenges in high throughput detection, countrywide sampling, and forensics in developing countries. Analyst 136: 3073-3082.

25. World Health Organization (WHO) (2005) Counterfeit \& substandard drugs - Frequently asked questions. Available: http://www.who.int/ medicines/services/counterfeit/faqs/en/. Accessed 11 March 2011.

26. World Health Organization (WHO) (2009) What are substandard medicines? Available: http:// www.who.int/medicines/services/counterfeit/ faqs/06/en/index.html. Accessed 6 January 2012.

27. World Health Organization (WHO) (2010) New definition for "substandard medicines". Working document QAS/10.344/Rev.1. Available: http://www.who.int/medicines/services/expert committees/pharmprep/14052010NewDefinition SubstandardMeds-QAS10-344Rev1.pdf. Accessed 6 January 2012.

28. World Health Organization (WHO) (2008) Counterfeit medical products. Executive Board 124 th session provisional agenda item 4.1 (EB124/14). Available: http://apps.who.int/gb/ ebwha/pdf_files/EB124/B124_14-en.pdf. Accessed 6 January 2012.

29. Sundar S, Sinha PR, Agrawal NK, Srivastava R, Rainey PM, et al. (1998) A cluster of cases of severe cardiotoxicity among kala-azar patients treated with a high-osmolarity lot of sodium antimony gluconate. Am J Trop Med Hyg 59: 139-143.

30. Rijal S, Chappuis F, Singh R, Boelaert M, Loutan L, et al. (2003) Sodium stibogluconate cardiotoxicity and safety of generics. Trans R Soc Trop Med Hyg 97: 597-598.

31. Boelaert M, Le Ray D, Van Der Stuyft P (2002) How better drugs could change kala-azar control. Lessons from a cost-effectiveness analysis. Trop Med Int Health 7: 955-959.

32. Newton PN, Green MD, Fernández FM, Day NPJ, White NJ (2006) Counterfeit antiinfective drugs. Lancet Infect Dis 6: 602-613.

33. Leslie T, Kaur H, Mohammed N, Kolaczinski K, Ord RL, et al. (2009) Epidemic of Plasmodium falciparum Malaria Involving Substandard Antimalarial Drugs, Pakistan, 2003. Emerg Infect Dis 15: $1753-1759$.

34. Caudron J-M, Ford N, Henkens M, Macé C, Kiddle-Monroe R, et al. (2008) Substandard medicines in resource-poor settings: a problem that can no longer be ignored. Trop Med Int Health 13: 1062-1072.

35. Newton PN, Fernández FM, Plançon A, Mildenhall DC, Green MD, et al. (2008) A collaborative epidemiological investigation into the criminal fake artesunate trade in South East Asia. PLoS Med 5: e32. doi:10.1371/journal.pmed.0050032.

36. Newton PN, Dondorp A, Green M, Mayxay M, White NJ (2003) Counterfeit artesunate antimalarials in southeast Asia. Lancet 362: 169.

37. Dondorp AM, Newton PN, Mayxay M, Van Damme W, Smithuis FM, et al. (2004) Fake antimalarials in Southeast Asia are a major impediment to malaria control: multinational cross-sectional survey on the prevalence of fake antimalarials. Trop Med Int Health 9: 1241-1246.

38. Newton PN, Amin AA, Bird C, Passmore P, Dukes G, et al. (2011) The primacy of public health considerations in defining poor quality medicines. PLoS Med 8: e1001139. doi:10.1371/ journal.pmed.1001139.

39. Ravinetto RM, Cloëz S, Scouflaire-Mallet S-M, Vandenbergh D (2009) Poor-quality medicines in developing countries. Lancet Infect Dis 9: 267-268.

40. World Health Organization (WHO) (2011) WHO Technical Report Series 961: WHO Expert Committee on Specifications for Pharmaceutical Preparations, Forty-fifth Report. Available: http://whqlibdoc.who.int/trs/WHO_ TRS_961_eng.pdf. Accessed 18 October 2011.

41. World Health Organization (WHO) (2010) Prequalification of Medicines Programme - a United Nations programme managed by the WHO. Available: http://apps.who.int/prequal/. Accessed 25 July 2011.

42. World Health Organization (WHO) (2011) 17th WHO Model List of Essential Medicines. Available: http://whqlibdoc.who.int/hq/2011/ a95053_eng.pdf. Accessed 24 January 2012.

43. Paladin Labs Inc (2010) Application for inclusion of miltefosine on WHO Model List of Essential Medicines. Available: http://www.who.int/ selection_medicines/committees/expert/18/ applications/Miltefosine_application.pdf. Accessed 13 December 2011.

44. Directorate General of Drug Registration, Government of the People's Republic of Bangladesh (2011) Directorate General of Drug Administration website. Available: http://www.dgda.gov. bd/. Accessed 18 October 2011. 